\title{
Neurocognitive changes in patients with schizophrenia during relapse and early remission
}

\author{
Nóra Balogh, MA \\ Dr. Anikó Égerházi, PhD \\ Dr. Roland Berecz, PhD* \\ Department of Psychiatry, University \\ of Debrecen, 98 Nagyerdei krt., \\ H-4012 Debrecen \\ HUNGARY
}

\begin{abstract}
Background and Objectives: Several studies have demonstrated that patients with schizophrenia have impaired cognitive functioning. In the literature there have been controversial results about the cognitive deficits occurring in the different states of the illness. Furthermore, there have been relatively few studies to investigate the associations between neurocognitive deficits and clinical status over time.

In order to follow the changes of neurocognitive subfunctions during relapse and early remission (clinically stable state), in the present study patients with schizophrenia were tested in the acute phase and in clinically stable state, and then the results were correlated with clinical symptoms.

Methods: Forty-two patients diagnosed with schizophrenia based on diagnostic interviews by clinicians and 43 normal controls were studied. Neurocognitive skills were evaluated with six subtests of the Cambridge Neuropsychological Test Automated Battery (CANTAB). Among patients with schizophrenia, symptom severity was assessed with the Positive and Negative Syndrome Scale (PANSS).

Results: Patients with schizophrenia performed significantly worse in all neurocognitive subtests compared to healthy controls at both relapse and clinically stable state. At follow-up these cognitive changes improved, however still marked dysfunctions were observed. The negative symptoms in the PANSS and CANTAB tests were negatively correlated with both results during relapse and clinically stable state.

Conclusions: Cognitive impairment exists among patients with schizophrenia compared to healthy subjects during both relapse and early remission suggesting that these deficits might be permanent.
\end{abstract}

Received: 27 April 2015

Revised: 10 July 2015

Accepted: 20 July 2015 


\section{Introduction}

Cognitive dysfunctions in schizophrenia have been increasingly regarded as a dominant part of the symptomatology ${ }^{1}$. Several studies have described that patients with schizophrenia are characterized by marked cognitive deficits, mainly in the areas of attention, visual memory, working memory and executive functions ${ }^{2,3}$. Their cognitive performance is consistently worse compared to healthy individuals in certain tests measuring neurocognitive functions ${ }^{4}$.

Longitudinally, schizophrenia is usually characterized by different episodes, i.e. periodic relapses and remission phases. During relapse, patients with schizophrenia show mainly specific psychotic symptoms, while the remission state is mainly characterized by milder and predominantly negative symptoms. This state is called "remission", rather than "recovery", because it fits more into the concept of schizophrenia due to the chronicity of the disorder. Unfortunately, remissions are usually time-limited periods in the course of schizophrenia, whereas recovery would mean complete convalescence from the illness ${ }^{5}$. Nevertheless, being in symptomatic remission does not mean that patients are doing completely well, since the remaining milder symptoms may negatively affect their functioning ${ }^{6}$.

Controversy exists in the literature, since in several studies patients in remission have shown improved executive functioning compared to those in relapse ${ }^{7-11}$. Moreover, another research found only weak correlation between improved symptoms and changes in cognitive dysfunctions ${ }^{12}$. These studies were characterized by a cross-sectional design. In several longitudinal studies it has been concluded that the neurocognitive performance of patients with schizophrenia is notably stable regardless of clinical state ${ }^{13,14}$.
Additionally, Heaton and co-workers ${ }^{15}$ found that cognitive deficits are trait-like characteristics of the disorder.

Furthermore, previously no significant differences in neurocognitive performance of patients with schizoaffective disorder between relapse and remission were reported, which suggests its trait-like nature ${ }^{16}$. In another study, patients with bipolar disorder also showed poorer neurocognitive performances regardless of their clinical state. Unfortunately it was a cross-sectional study, thus a longitudinal designed study may help to investigate whether cognitive impairments are trait or state-like characteristics of bipolar disorder ${ }^{17}$.

Results of previous longitudinal studies suggest that antipsychotic medications have only a moderate effect on the cognitive skills of patients $^{18,19}$; thus, cognitive deficits seem to be largely independent of antipsychotic treatment ${ }^{20}$ and are present in all states -prodromal, relapse and remission- of the disorder ${ }^{21}$. Nevertheless, Knowles and co-workers ${ }^{22}$ in a meta-analysis concluded that processing speed impairment was affected by antipsychotic medications, while other cognitive tasks were not.

The main aim of the present study was to examine cognitive dysfunctions in patients with schizophrenia at two different time points: first in an acute phase (when patients presented predominantly positive psychotic symptoms) and at a second time in early remission (clinically stable state) in order to evaluate specific changes in their cognitive skills at these two stages using a language-independent, neurocognitive battery to measure specific cognitive subfunctions. Furthermore, specific neurocognitive deficits were measured in relation to clinical status and symptoms. Our second aim was to compare the cognitive performance of patients in early remission with that of healthy individuals. 


\section{Subjects and methods}

\section{Subjects}

Forty-two patients (18 women, 24 men mean age $39 \pm 10$ years) with schizophrenia (the majority had the paranoid type, while 4 were hebephrenic) meeting DSM-IV criteria $^{23}$ participated in our study. All patients had previous psychotic episodes. The average duration of illness was 15 years $(\mathrm{SD}=10.1)$. All of them were hospitalized during the testretest interval (Table 1.).

Table 1

Demographic and clinical characteristics of patients and healthy subjects ( $\mathrm{p}>0.05$, not significant).

Schizophrenic patients

\begin{tabular}{lcc}
\hline Sex (male/female) & $24 / 18$ & $20 / 23$ \\
Age (yrs.) & $39 \pm 10$ & $37 \pm 9$ \\
Education (yrs.) & 13.70 & 15.80 \\
Age of onset (yrs.) & 26.40 & - \\
\hline
\end{tabular}

They were recruited from the inpatient unit of the Department of Psychiatry, University of Debrecen, Hungary and at the time of the first examination were in the acute phase of the disorder. PANSS had to be scored with a symptom severity of $\geq 4$ points ("medium" or more severe): a) delusions; b) unusual thought content; c) hallucinatory behaviour; d) blunted affect; e) passive/apathetic social withdrawal. Exclusion criteria were any kind of severe physical illness, drug or alcohol addiction and schizoaffective disorders. The research diagnosis was based on diagnostic interviews guided by clinicians independent of the study. These interviews included the Structured Clinical Interview for DSM Disorders - Clinician Version (SCID$\mathrm{CV}$ ), the Research Version (SCID-RV) and also the Positive and Negative Syndrome Scale (PANSS) was evaluated. The treatment of the patients were as follows: amisulpride: 2 patients, aripiprazole: 5 , clozapine: 10 , flupenthixol: 2, haloperidol: 3, olanzapine: 11 , paliperidone: 8 , quetiapine: 9 , risperidone: 6 , ziprasidone: 1, benzatropine: 10 , biperiden: 1 , procyclidine: 1 and clonazepam: 1 patient. This medication therapy remained unchanged throughout the test-retest interval.

Forty-three age-matched healthy control individuals (23 women, 20 men - mean age 37 \pm 9 years) were also assessed. These individuals had never met the criteria for any kind of psychiatric disorder in their course of life. None of these subjects had any substance abuse, which could influence their cognitive performance. They were informed properly about the aim of the study and the details of the examination and they consented to participate.

This study was approved by the Regional and Institutional Ethics Committee of University of Debrecen, Medical and Health Science Center (UDMHSC REC/IEC) and was conducted in accordance with The Code of Ethic of the World Medical Association (Declaration of Helsinki) for experiments involving humans. Some of the data were gained from the SCHIZOBANK - a national schizophrenia biobank - database (e.g. PANSS scores, age, gender, drug administration), since 
patients were tested while this biobank had been being built ${ }^{24}$. The main demographic and clinical characteristics of patients and healthy subjects are shown in Table 1.

\section{Methods}

Patients and healthy subjects were tested with the Cambridge Neuropsychological Test Automated Battery (CANTAB), a computerized neuropsychological test battery which allows assessing several cognitive subfunctions in a standardized way. It is a more comprehensive neuropsychological test battery than those single neurocognitive tasks which can measure only specific cognitive functions of the brain. The CANTAB database consists of 3,000 healthy control persons with different gender, age and IQ. During validation no significant difference was found in the cognitive performance between the Hungarian healthy volunteers and the database of CANTAB. Therefore, it was assumed that CANTAB was a valid measure for the assessment of cognitive skills in the Hungarian population ${ }^{25}$.

\section{Assessment of cognitive functions} (CANTAB)

Several tests of CANTAB are useful to demonstrate cognitive impairment in patients with schizophrenia in tasks measuring working memory, visual and spatial memory, executive functions, sustained attention, information processing, formula recognition and deficit in the ability of learning new information $^{26-28}$. CANTAB is suitable for individuals who have never used a computer with a touch screen and a hand switch. The first test of CANTAB (Motor Screening - MOT) was used to assure that the participants had the physical and mental abilities necessary to complete the test. Technical details of the tests can be found at the following website: (www.camcog.com, Cambridge Cognition).
In our study the neurocognitive functions of the schizophrenic patients and healthy individuals were tested with six subsets of CANTAB, which were brief enough for the patients to complete. Our selection was based on a previous study conducted at our Department, which showed that prepsychotic patients and schizophrenic patients with predominantly negative symptoms performed significantly worse on these tasks compared to healthy controls ${ }^{29}$. Therefore, the present study was focused on these domains. However, other aspects of cognition, such social cognition, of the same patient sample were reported by our group previously ${ }^{30}$. Thus, the following tests were administered to the participants:

The tests measuring visual memory were the Paired Associate Learning (PAL) and the Spatial Recognition Memory (SRM) subtests. The PAL subtest requires participants to learn new specific visual patterns and locate them on the computer screen. The SRM subtest estimates the ability of patients to remember the spatial location of visual stimuli. For the evaluation of sustained attention, Rapid Visual Information Processing (RVP) was administered. The tests measuring working memory and planning skills were the Spatial Working Memory (SWM) and the Stockings of Cambridge (SOC) tasks. The SWM subtest measures how the subject can keep spatial information in the working memory and it also shows the searching strategy used by the subject. The SOC subtest is analogous with the "Tower of London" test; it is suitable for evaluating the spatial problem solving and planning abilities ${ }^{31}$.

An assistant who was trained to use CANTAB gave instructions for each task. Participants were asked to carry out the tasks under the assistant's guidance.

\section{Psychotic symptom severity}

The PANSS (Positive and Negative Syndrome Scale) was used to assess symptom 
severity among patients ${ }^{32}$ and it was administered by a trained psychologist, who was the same for all the patients.

\section{Study design}

Patients were examined twice - first in an acute phase (after admission as soon as they were able to cooperate, on average within 4 days) and at a second time in a clinically stable state. The early remission or clinically stable state was defined as being discharged by the clinician from the acute ward within a maximum of 3 months. The mean period of time between assessments was 31 days $(\mathrm{SD}=32.5)$. When a patient with schizophrenia in the acute phase was admitted to the acute ward, a trained psychologist conducted the semi-structured interview of PANSS and then the patient was tested with CANTAB in a laboratory room. After some time -based on the therapist's judgement- when the patient recovered from the episode and entered into clinically stable state, PANSS and CANTAB were repeated before discharge. The PANSS scores of positive symptoms decreased by $18 \%$, the negative symptoms by $12 \%$, the general symptoms by $17 \%$ and the overall scores by $16 \%$. Healthy individuals were tested only once.

Patients have been divided into subgroups according to the criteria used by Braw and co-workers ${ }^{33}$ based on their PANSS scores: (1) Positive and general symptomatic remission (PSR) was defined as $\leq 3$ on the following items: P1 (Delusions), P2 (Conceptual disorganisation), P3 (Hallucinatory behaviour), G5 (Mannerism and posturing) and G9 (Unusual thought content). (2) Negative symptomatic remission (NSR) was defined as $\leq 3$ on items N1 (Blunted affect), N4 (Social withdrawal) and N6 (Lack of spontaneity) ${ }^{30}$.

At baseline, PANSS scores were 21.8 (SD $=4.8)$ for positive symptoms, $23.5(\mathrm{SD}=5.6)$ for negative symptoms, $44.3(\mathrm{SD}=9.4)$ for general symptoms and $89.8(\mathrm{SD}=15.6)$ for overall scores. At clinically stable state, PANSS scores were $17.7(\mathrm{SD}=4.8)$ for positive symptoms, $20.8(\mathrm{SD}=4.8)$ for negative symptoms, $36.8(\mathrm{SD}=8.9)$ for general symptoms and $75.2(\mathrm{SD}=15.5)$ for overall scores.

\section{Statistical data analysis}

The PANSS scores were calculated based on the total points in the negative, positive and general pathology domain. Participants' scores in all CANTAB subtest results were calculated from median scores on the basis of the CANTAB internal normative database of healthy volunteers. Since we could not assume normal distribution in all tests, the scores of the patients and healthy individuals were compared using non-parametric Mann-Whitney t-test. Correlation between the PANSS scores and CANTAB subtest results was carried out by Spearman's non-parametric tests. The CANTAB scores of patients in acute and in clinically stable phase were compared by the non-parametric Mann-Whitney t-test. Statistical calculations were carried out using the GraphPad Prism 6.00 for Windows software (GraphPad Software, San Diego, CA, USA, http://www.graphpad.com) and $\mathrm{p}<0.05$ was considered as significant.

\section{Results}

\section{CANTAB test results}

Patients showed significantly worse performance in all CANTAB tests (SRM, PAL, SWM, SOC, RVP) compared to healthy controls in both states (relapse and clinically stable state) (Figure 1). There was no statistically significant difference between the two states with regard to neurocognitive tasks, although the PAL test showed a weak tendency for improvement $(\mathrm{p}=0.2)$ (Figure 2). 


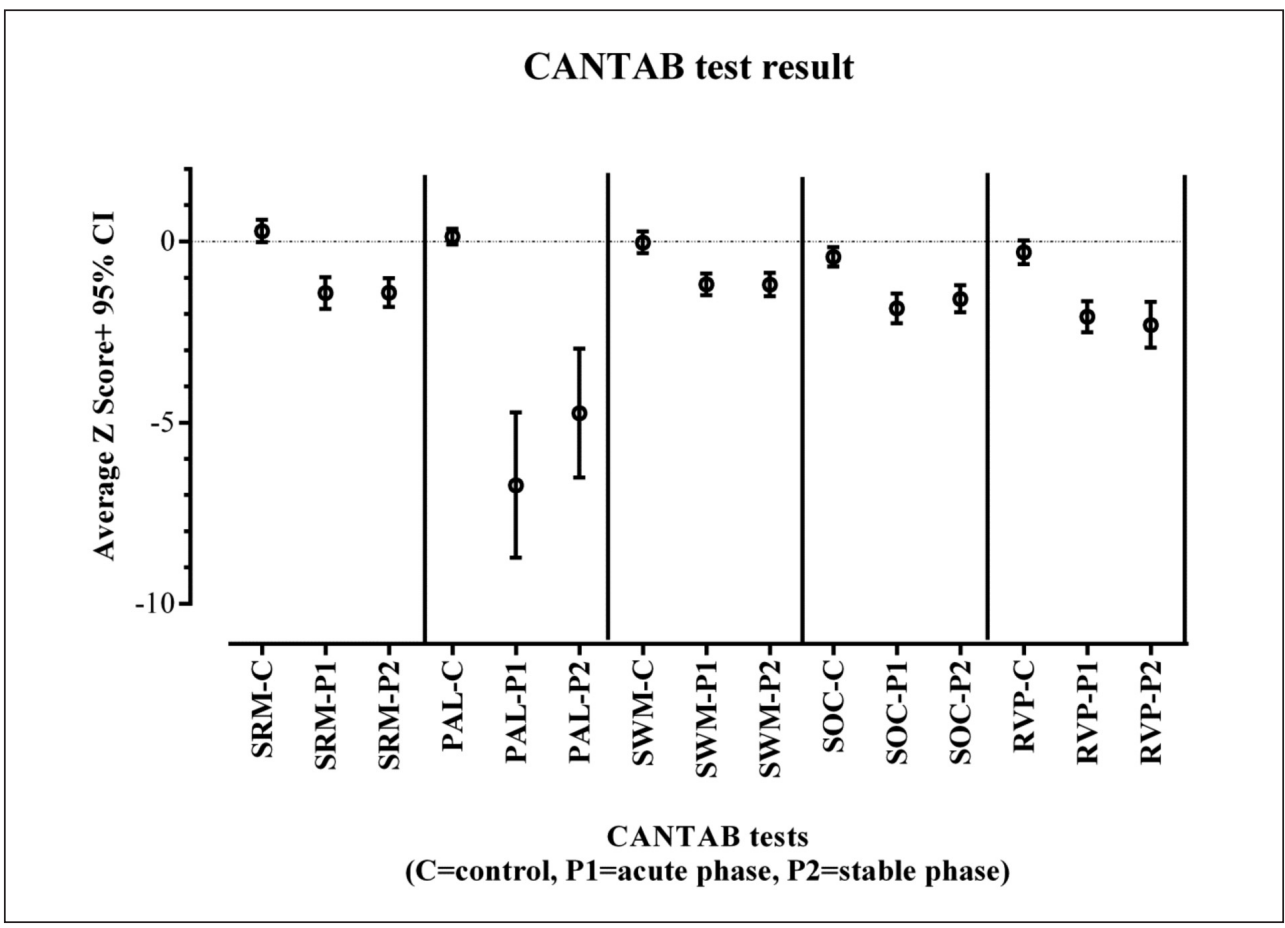

Figure 1. CANTAB tests average results (+95\% confidence interval, CI) in healthy volunteers and schizophrenic patients $(\mathrm{C}=$ control, $\mathrm{P} 1=$ acute phase, $\mathrm{P} 2=$ remission phase CANTAB tests: $\mathrm{SRM}$ - Spatial Recognition Memory,

PAL - Paired Associate Learning, SWM - Spatial Working Memory, SOC - Stockings of Cambridge, RVP - Rapid Visual Information Processing).

\section{PANSS and CANTAB tests during relapse}

The overall sum of the positive symptoms of PANSS were correlated with the performance of patients in the SWM subtest (measuring spatial working memory, Z-score $=-1.1$, $\mathrm{p}<0.05)$ in acute phase. Negative symptoms were correlated with visual spatial memory and paired association learning (SRM, Zscore $=-1.4$, PAL, Z-score $=-6.7 ; \mathrm{p}<0.05$ ) (Figure 1). General symptoms of PANSS also correlated with the PAL (paired associated learning) during relapse (Table 2).

\section{PANSS and CANTAB tests during clinically stable state}

There was no correlation between the positive symptoms of PANSS and any of the subtests in clinically stable state $(\mathrm{p}>0.05$, Table 2).

The negative symptoms of PANSS were significantly correlated with paired associate learning and visual spatial memory functions (PAL, SRM) (Figure 2).

There was also a significant correlation between the general symptoms and the PAL (paired associated learning) in clinically stable state (Table 3). 


\section{PAL-PANSS negative symptoms:acute phase}

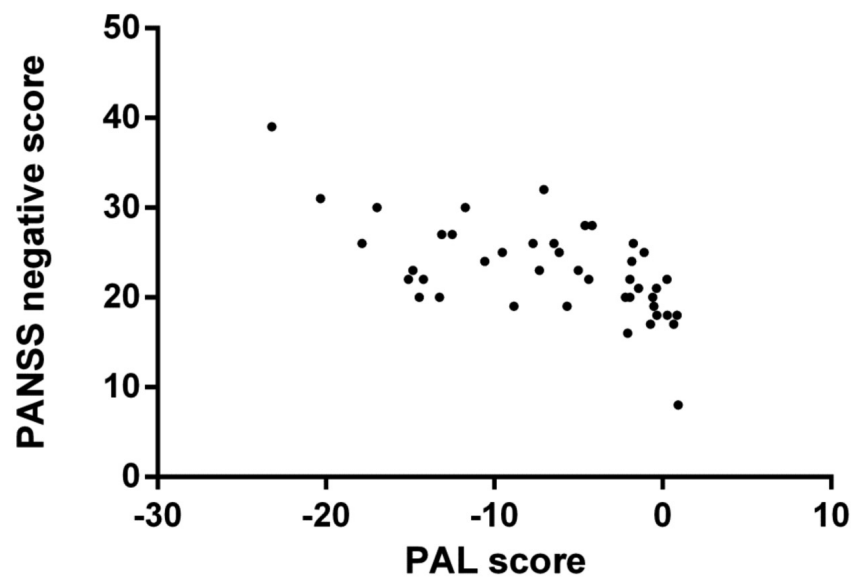

PAL-PANSS negative symptoms: early remission

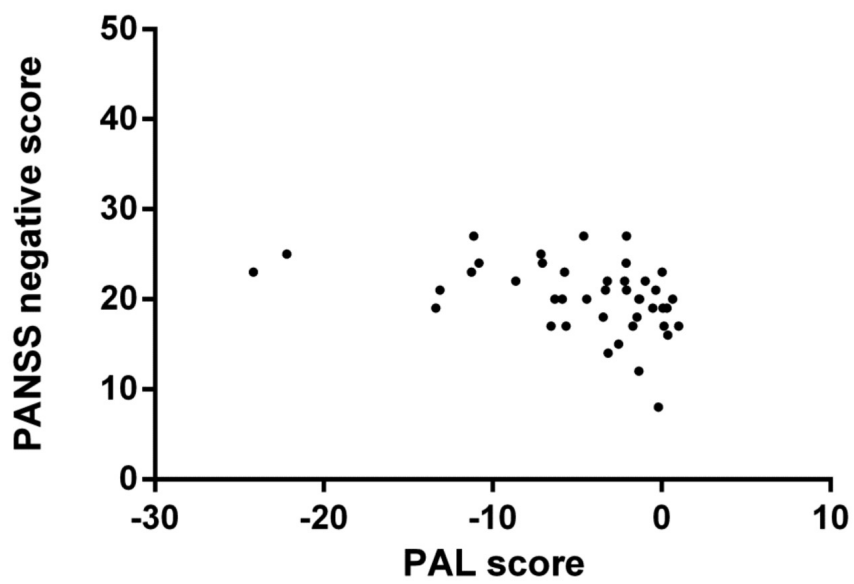

Figure 2. Correlation between PANSS negative symptoms and the

Paired Associate Learning (PAL) test in acute phase and in early remission. 
Table 2

Correlation between PANSS negative, positive and general symptoms in schizophrenic patients during acute phase (Spearman $r$ and 95\% confidence intervals) and cognitive subfunctions.

\begin{tabular}{lccccc} 
& SRM & PAL & SWM & SOC & RVP \\
\hline Positive symptoms & 0.02 & -0.06 & $0.38^{*}$ & -0.08 & -0.07 \\
& $(-0.29 / 0.32)$ & $(-0.37 / 0.25)$ & $(0.08 / 0.62)$ & $(-0.38 / 0.24)$ & $(-0.41 / 0.29)$ \\
\hline Negative symptoms & $-0.43^{*}$ & $-0.61^{* *}$ & -0.12 & -0.11 & 0.03 \\
& $(-0.65 /-0.14)$ & $(-0.77 /-0.37)$ & $(-0.42 / 0.20)$ & $(-0.41 / 0.21)$ & $(-0.32 / 0.38)$ \\
\hline General symptoms & -0.12 & $-0.40 / *$ & -0.01 & -0.16 & -0.10 \\
& $(-0.42 / 0.19)$ & $(-0.63 /-0.11)$ & $(-0.32 / 0.30)$ & $(-0.45 / 0.16)$ & $(-0.44 / 0.26)$ \\
\hline
\end{tabular}

$* \mathrm{p}<0.05, * * \mathrm{p}<0.001$.

SRM: Spatial Recognition Memory, PAL: Paired Associate Learning, SWM: Spatial Working Memory, SOC: Stockings of Cambridge, RVP: Rapid Visual Processing.

Table 3

Correlation between PANSS negative, positive and general symptoms in schizophrenic patients during early remission (Spearman $\mathrm{r}$ and 95\% confidence intervals).

\begin{tabular}{lccccc} 
& SRM & PAL & SWM & SOC & RVP \\
\hline Positive symptoms & 0.02 & -0.04 & 0.14 & 0.03 & -0.04 \\
& $(-0.33 / 0.29)$ & $(-0.35 / 0.27)$ & $(-0.17 / 0.44)$ & $(-0.29 / 0.33)$ & $(-0.28 / 0.35)$ \\
\hline Negative symptoms & $-0.47^{*}$ & $-0.47 *$ & -0.01 & -0.18 & -0.17 \\
& $(-0.68 /-0.18)$ & $(-0.68 /-0.19)$ & $(-0.31 / 0.30)$ & $(-0.47 / 0.14)$ & $(-0.47 / 0.16)$ \\
\hline General symptoms & -0.22 & $-0.35 / *$ & -0.03 & -0.17 & -0.08 \\
& $(-0.49 / 0.10)$ & $(-0.60 /-0.05)$ & $(-0.28 / 0.34)$ & $(-0.46 / 0.14)$ & $(-0.25 / 0.39)$ \\
\hline
\end{tabular}

$* \mathrm{p}<0.05$.

SRM: Spatial Recognition Memory, PAL: Paired Associate Learning, SWM: Spatial Working Memory, SOC: Stockings of Cambridge, RVP: Rapid Visual Processing.

\section{Discussion}

As with previous studies, the results of this study show that patients with schizophrenia have cognitive impairments compared to healthy people even during in clinically stable state $^{34,35}$. Decreased performance was observed in visual spatial tasks, working memory and planning and also in sustained attention. The Paired Associate Learning task was confirmed to be a very sensitive for cognitive impairment ${ }^{3,29}$. These cognitive changes are related to temporal, parietal lobe and frontostriatal dysfunctions, as well as abnormal function of the right prefrontal lobe among patients with schizophrenia.

When comparing cognitive functions during relapse (acute phase) and in clinically stable state, no statistical difference in cognitive skills was found between the two states. This may suggest that the cognitive dysfunctions are persistent in all phases of the disorder and may represent the core symptom of the disease. 
In our study the maximum time interval between the two testing was 3 months and confirms the findings of previous studies, which have found that cognitive skills are stable over time in schizophrenia ${ }^{13,36,37}$. The relative stability of cognitive impairments can be emphasized; however, a moderate cognitive improvement may exists, as some papers reported longitudinal improvements in memory and executive functioning after 5 years ${ }^{38}$, or 20 years after index hospitalization ${ }^{39}$.

Limitations of the study are that only a relatively small sample was tested. Furthermore, the time interval between test and retest was also short due to our definition of an early remission or clinically stable state, which was less stringent then remission. The control group was tested once, which might cause a test-retest issue, although stability of their performance is expected over time due to the disposition of the test.

In conclusion, we have found evidence that there are cognitive differences between patients and healthy individuals in both relapse and in clinically stable state and this finding is consistent with previous literature data. The lack of significant improvement in neurocognitive functions in the present short-term follow-up study suggests that these dysfunctions are persistent and may be considered as the core symptom of the disorder. Future studies with longer follow-up periods may provide further insight in the cognitive changes during the course of schizophrenia.

\section{Acknowledgement}

The authors would like to thank Mikéné Almási Ildikó for carrying out the CANTAB assessments.

\section{Conflict of interest}

The authors declare that they have no competing interests.

\section{Financial support}

The study was supported for BN by SCHIZO-08 Consortium (University of Debrecen, Hungarian Society of Clinical Neurogenetics (HSCN) for providing data, Gedeon Richter Plc., UD-GenoMed Medical Genomic Technologies Ltd., Kripto Ltd., TÁMOP-4.2.1/B09/1/KONV-2010-0007 for unrestricted financial support of the study for all authors.

\section{References}

1. Harvey PD. Cognition and the differential diagnosis of schizophrenia. World Psychiatry 2008; 7(1): 30-2.

2. Pantelis C, Barnes TR, Nelson HE Tanner S, Weatherley L, Owen AM, et al. Frontal-striatal cognitive deficits in patients with chronic schizophrenia. Brain. 1997; 120(10): 1823-43.

3. Ferencz A, Bartók E, Berecz R, Glaub T, Degrell I. [Cognitive functions in prepsychotic and negative-symptom schizophrenic patients]. Neuropsychopharmacol Hung. 2005; 7(2): 61-5.

4. Yun Da Y, Hwang SS, Kim Y, Lee YH, Kim YS, Jung HY. Impairments in executive functioning in patients with remitted and non-remitted schizophrenia. Prog Neuropsychopharmacol Biol Psychiatry. 2011; 35(4): 1148-54.

5. Davidson L, Schmutte T, Dinzeo T, Andres-Hyman R. Remission and recovery in schizophrenia: Practitioner and patient perspectives. Schizophr Bull. 2008; 34(1): 5-8.

6. Lambert M, De Marinis T, Pfeil J, Naber D, Schreiner A. Establishing remission and good clinical functioning in schizophrenia: Predictors of best outcome with long-term risperidone long-acting injectable treatment. Eur Psychiatry. 2010; 25(4): 220-9. 
7. Joyce E, Hutton S, Mutsatsa S, Gibbins H, Webb E, Paul $\mathrm{S}$, et al. Executive dysfunction in first-episode schizophrenia and relationship to duration of untreated psychosis: The West London Study. Br J Psychiatry Suppl. 2002; 43: S38-S44.

8. Rund BR, Melle I, Friis S, Larsen TK, Midbøe LJ, Opjordsmoen $\mathrm{S}$, et al. Neurocognitive dysfunction in firstepisode psychosis: Correlates with symptoms, premorbid adjustment, and duration of untreated psychosis. Am J Psychiatry. 2004; 161(3): 466-72.

9. Helldin L, Kane JM, Karilampi U, Norlander T, Archer T. Remission and cognitive ability in a cohort of patients with schizophrenia. J Psychiatr Res. 2006; 40(8): 738-45.

10. Brissos S, Dias VV, Balanzá-Martinez V, Carita AI, Figueira ML. Symptomatic remission in schizophrenia patients: Relationship with social functioning, quality of life, and neurocognitive performance. Schizophr Res. 2011; 129(2-3): 133-6.

11. Stratta P, Rossi A. Short-term remission in schizophrenia as a combination of several outcome measures. Psychiatry Res. 2013; 209(3): 401-5.

12. Szöke A, Trandafir A, Dupont ME, Méary A, Schürhoff F, Leboyer M. Longitudinal studies of cognition in schizophrenia: Meta-analysis. Br J Psychiatry. 2008; 192(4): 248-57.

13. Keefe RS, Sweeney JA, Gu H, Hammer RM, Perkins DO, McEvoy JP, et al. Effects of olanzapine, quetiapine, and risperidone on neurocognitive function in early psychosis: A randomized, double-blind 52-week comparison. Am J Psychiatry. 2007; 164(7): 1061-71.

14. Keefe RS, Fenton WS. How should DSM-V criteria for schizophrenia include cognitive impairment? Schizophr Bull. 2007; 33(4): 912-20.

15. Heaton RK, Gladsjo JA, Palmer BW, Kuck J, Marcotte TD, Jeste DV. Stability and course of neuropsychological deficits in schizophrenia. Arch Gen Psychiatry. 2001; 58(1): 24-32.

16. Madre M, Radua J, Landin-Romero R, Alonso-Lana $\mathrm{S}$, Salvador R, Panicali F, et al. Trait or state? A longitudinal neuropsychological evaluation and fMRI study in schizoaffective disorder. Schizophrenia Research. 2014; 159(2-3): 458-64.

17. Martínez-Arán A, Vieta E, Reinares M, Colom F, Torrent C, Sánchez-Moreno J, Benabarre A, et al. Cognitive function across manic or hypomanic, depressed, and euthymic states in bipolar disorder. Am J Psychiatry. 2004; 161(2): 262-70.

18. Hill SK, Bishop JR, Palumbo D, Sweeney JA. Effect of second-generation antipsychotics on cognition: Current issues and future challenges. Expert Rev Neurother. 2010; 10(1): 43-57.
19. Censits DM, Ragland JD, Gur RC, Gur RE. Neuropsychological evidence supporting a neurodevelopmental model of schizophrenia: A longitudinal study. Schizophr Res. 1997; 24(3): 289-98.

20. Keefe RS. Should cognitive impairment be included in the diagnostic criteria for schizophrenia? World Psychiatry. $2008 ; 7(1): 22-8$.

21. Green MF, Kern RS, Heaton RK. Longitudinal studies of cognition and functional outcome in schizophrenia: Implications for MATRICS. Schizophr Res. 2004; 72(1): 41-51.

22. Knowles EE, David AS, Reichenberg A. Processing speed deficits in schizophrenia: Reexamining the evidence. Am J Psychiatry. 2010; 167(7): 828-35.

23. American Psychiatric Association. Diagnostic and Statistical Manual of Mental Disorders. 4th ed. Washington DC; 1994.

24. Inczédy-Farkas G, Benkovits J, Balogh N, Almos P, Scholtz B, Zahuczky G, et al. [SCHIZOBANK - The Hungarian national schizophrenia biobank and its role in schizophrenia research and in personalized medicine]. Orv Hetil. 2010; 151(35): 1403-8.

25. Bartók E, Berecz R, Glaub T, Degrell I. Számítógépes neurokognitív vizsgálati programcsomag magyarországi validálása. [Validation of the computerized neurocognitive test battery [CANTAB] in Hungary.] Psychiatr Hung. 2001; 16(2): 121-30.

26. Hutton SB, Puri BK, Duncan LJ, Robbins TW, Barnes TR, Joyce EM. Executive function in first-episode schizophrenia. Psychol Med. 1998; 28(2): 463-73.

27. Prouteau A, Verdoux H, Briand C, Lesage A, Lalonde $\mathrm{P}$, Nicole $\mathrm{L}$, et al. The crucial role of sustained attention in community functioning in outpatients with schizophrenia. Psychiatry Res. 2004; 129(2): 171-7.

28. Tyson PJ, Laws KR, Roberts KH, Mortimer AM. Stability of set-shifting and planning abilities in patients with schizophrenia. Psychiatry Res. 2004; 129(3): 229-39.

29. Bartók E, Berecz R, Glaub T, Degrell I. Cognitive functions in prepsychotic patients. Prog Neuropsychopharmacol Biol Psychiatry 2005; 29(4): 621-5.

30. Balogh N, Égerházi A, Berecz R, Csukly G. Investigating the state-like and trait-like characters of social cognition in schizophrenia: A short term follow-up study. Schizophr Res. 2014; 159(2-3): 499-505.

31. Sweeney JA, Kmiec JA, Kupfer DJ. Neuropsychologic impairments in bipolar and unipolar mood disorders on the CANTAB neurocognitive battery. Biol Psychiatry. 2000; 48(7): 674-84.

32. Kay SR, Fiszbein A, Opler LA. The positive and negative syndrome scale (PANSS) for schizophrenia. Schizophr Bull. 1987; 13(2): 261-76. 
33. Braw Y, Benozio A, Levkovitz Y. Executive functioning during full and partial remission (positive and negative symptomatic remission) of schizophrenia. Schizophr Res. 2012; 142(1-3): 122-8.

34. Šoštarič M, Zalar B. The overlap of cognitive impairment in depression and schizophrenia: A comparative study. Psychiatr Danub. 2011; 23(3): 251-6.

35. Veltro F, Mazza M, Vendittelli N, Alberti M, Casacchia M, Roncone R. A comparison of the effectiveness of problem solving training and of cognitive-emotional rehabilitation on neurocognition, social cognition and social functioning in people with schizophrenia. Clin Pract Epidemiol Ment Health. 2011; 7: 123-32.

36. Hyde TM, Nawroz S, Goldberg TE, Bigelow LB, Strong D, Ostrem JL, et al. Is there cognitive decline in schizophrenia? A cross-sectional study. Br J Psychiatry. 1994; 164(4): 494-500.

37. Russell AJ, Munro JC, Jones PB, Hemsley DR, Murray $\mathrm{RM}$. Schizophrenia and the myth of intellectual decline. Am J Psychiatry. 1997; 154(5): 635-9.
38. Gold S, Arndt S, Nopoulos P, O'leary DS, Andreasen NC. Longitudinal study of cognitive function in first-episode and recent-onset schizophrenia. Am J Psychiatry. 1999; 156(9): 1342-8.

39. Bonner-Jackson A, Grossman LS, Harrow M, Rosen C. Neurocognition in schizophrenia: A 20-year multi-followup of the course of processing speed and stored knowledge. Compr Psychiatry. 2010; 51(5): 471-9.

\footnotetext{
* Corresponding author:

Dr. Roland Berecz

Department of Psychiatry

University of Debrecen

98 Nagyerdei krt.

H-4012 Debrecen

Hungary

Tel: +36305659310

E-mail: rberecz@med.unideb.hu
} 